\title{
Ueber eine Titration von Hydrargyrum praecipitatum alb.
}

\author{
Von E. Rupp. \\ (Eingegangen den 27. VII. 1903.)
}

Ein in Fachzeitschriften des öfteren behandelter Gegenstand ist die Analyse des weißen Präzipitates, für den eine quantitative Gehaltbestimmung rücksichtlich der mit der Darstellungsweise wechselnden Zusammensetzung, in der Tat nicht unangebracht erscheint.

Ich möchte $z u$ diesem $Z$ wecke eine Titration in Vorschlag bringen, die mit dem analytischen Hilfsmaterial des Arzneibuches ohne Um. ständlichkeit durchtührbar ist, und ergänzt durch die an alle Quecksilberverbindungen gestellte Forderung vollkommener Flüchtigkeit, ein ausreichendes Kriterium für ein pharmakopoegerechtes Präparat sein dürfte.

Eine abgewogene Substanzmenge wird mit Salpetersäure umgesetzt zu Merkurinitrat, Ammonnitrat und Salzsäure, sodann durch ein bekanntes Volum überschüssiger $5 / 10$ Silberlösung die der Quecksilber. titration hinderliche Salzsäure gefällt und das Ganze mit n/10 Rhodan titriert, wobei das gebildete Merkurinitrat und das im Ueberschuß zugesetzte Silbernitrat in unlösliche und neben einander bestimmbare Rhodanide übergehen ${ }^{1}$ ). Es resultiert also die Rhodanverbrauchsmenge aus der im Untersuchungsmaterial vorhanden gewesenen Quecksilberund Chlormenge. Der Rhodanverbrauch für Quecksilber ist direkt proportional der Hg-Menge, der Rhodanwert für Chlor ist umgekehrt proportional der Cl-Menge, denn je mehr Chlor vorhanden gewesen, um so weniger zurücktitrierbares Silbernitrat wird im Ueberschuß verbleiben. Hiernach möchte es zunächst scheinen, daß auch bei unreinen oder unrichtig zusammengesetzten Präzipitatsorten durch gegenseitige Kompensation ein richtiger Rhodansummenwert zu stande kommen könnte. Eine Betrachtung der stöchiometrischen Verhältnisse zeigt, jedoch, daß die wechselseitigen Beziehungen zwischen $\mathrm{Hg}$ und $\mathrm{Cl}$ Aenderungen der Rhodanwerte in gleichem Sinne veranlassen müssen, denn, enthält eine bestimmte Gewichtsmenge des zu untersuchenden Präparates zu viel $\mathrm{Hg}$, - großer Rhodanwert, so wird es um so weniger Cl enthalten, - großer Rhodanwert.

Enthält es umgekehrt zu viel Cl, - kleiner Rhodanwert, so wird es zu wenig $\mathrm{Hg}$ enthalten, - kleiner Rhodanwert. Hierbei bleibt es sich gleichgültig ob das $\mathrm{Cl}$ im Moleküle des Präparates selbst oder in

1) Berl. Ber. 35, 2015. 
einer fremden Beimischung sitzt, als welche Chlorammon und Merkurichlorid in Betracht kommen.

Die Ausführung der Analyse ist die folgende: 0,2 $\mathrm{g}$ Präzipitat übergießt man in einem Kochkölbchen mit $25 \mathrm{ccm}$ offizineller $25 \%$ iger Salpetersäure und erhitzt lose verschlossen 5 Minuten lang auf dem Wasserbade um vollständige Lösung herbeizuführen.

$$
\mathrm{Hg}<\mathrm{Cl} \mathrm{NH}_{2}+3 \mathrm{H} \mathrm{NO}_{8}=\mathrm{Hg}\left(\mathrm{NO}_{8}\right)^{2}+\mathrm{NH}_{4} \mathrm{NO}_{3}+\mathrm{HCl} .
$$

Man fügt dann unter Umschwenken $10 \mathrm{~cm}$ n/10 Silberlösung hinzu und spült mit ca. $5 \mathrm{ccm}$ Wasser nach, worauf nochmals 10 Minuten weiter erhitzt wird. Die Lösung erscheint dann vollkommen klar, während das ausgeschiedene Chlorsilber in dichtflockiger Form zu Boden sitzt. Nach dem Erkalten macht man einen Zusatz von ca. $5 \mathrm{ccm}$ $10 \%$ iger Eisenalaunlösung und titriert mit $\mathbf{n} / 10$ Rhodan auf deutlichen Eintritt der Indikatorreaktion.

$$
\begin{aligned}
& \mathrm{Hg}<\mathrm{NO}_{3}+2 \mathrm{NH}_{4} \cdot \mathrm{CNS}=\mathrm{Hg}<{ }_{\mathrm{CNS}}^{\mathrm{CNS}}+2 \mathrm{NH}_{4} \cdot \mathrm{NO}_{3}, \\
& \mathrm{AgNO} \mathrm{N}_{3}+\mathrm{NH}_{4} \cdot \mathrm{CNS}=\mathrm{AgCNS}+\mathrm{NH}_{4} \mathrm{NO}_{8} \text {. } \\
& \underbrace{1 \mathrm{Hg} \cdot \mathrm{NH}_{2} \cdot \mathrm{Cl}}_{251,8 \mathrm{~g}}=\underbrace{1 \mathrm{AgNO}}_{\text {für } \mathrm{Cl}}+\underbrace{2 \mathrm{NH}_{4} \mathrm{CNS}}_{\text {für }} \\
& \text { daher } 0,02518 \mathrm{~g} \text { Präzipitat }=1 \mathrm{ccm} \text { n/10 } \mathrm{Ag} \mathrm{NO}_{8} \\
& 0,01259, n=1, n / 10 \text { Rhodan und }
\end{aligned}
$$

$0,2 \mathrm{~g}$ Präzipitat $=7,95 \mathrm{~cm} \mathrm{n} / 10 \mathrm{AgNO}$ n/10 Rhodan.

Zurückzutitrierende $\mathrm{Ag} \mathrm{NO}$-Menge $=10-7,95=2,05 \mathrm{ccm}=2,05 \mathrm{ccm}$

Rhodangesamtverbrauch $15,89+2,05=17,94 \mathrm{ccm}$.

Ein in dieser Weise titriertes Präparat beanspruchte pro $0,2 \mathrm{~g}$ in mehreren Titrationen je $17,8 \mathrm{ccm}$ Rhodan.

Bei der gravimetrischen Quecksilberbestimmung lieferten $0,3 \mathrm{~g}=0,2724 \mathrm{~g}$ $\mathrm{HgS}=98,41 \%$ des berechneten Wertes von 0,2768 g.

Der berechnete Gesamtrhodanwert für das analysierte Präparat pro 0,2 g Substanz $=0,19682 \mathrm{~g}$ Präzipitat betrüge somit $17,76 \mathrm{ccm}$, $\left(15,64 \mathrm{ccm}\right.$ für $\mathrm{Hg}, 2,12$ für unverbrauchtes $\mathrm{Ag} \mathrm{NO} \mathrm{O}_{3}$ ).

Die nahe Uebereinstimmung des gefundenen Rhodanwertes von $17,8 \mathrm{ccm}$ mit dem berechneten von $17,76 \mathrm{ccm}$ zeigt an, daß die restierenden 1,59\% jedenfalls nicht aus Salmiak bestanden, da sich in diesem Falle der Silbernitratverbrauch um 0,59 ccm erhöht, also der Rhodanwert um $0,59 \mathrm{ccm}$ verringert haben müßte und $17,21 \mathrm{ccm}$ betragen hätte.

Die Berechnung zeigt, wie deutlich sich gerade diese wichtigste Verunreinigung im Resultate $z \mathfrak{u}$ erkennen gibt.

Pro praxi dürfte der tür obige Mengenverhältnisse zu fordernde Rhodanverbrauch auf 17,7-18,0 com festzulegen sein.

Chem. Univers.-Laborat. (Ph. Abt.) Freiburgi. B. 